\title{
The rapid and visual detection of methicillin-susceptible and methicillin- resistant Staphylococcus aureus using multiplex loop-mediated isothermal amplification linked to a nanoparticle- based lateral flow biosensor
}

\author{
Xu Chen ${ }^{1,2,3}$, Kai Ma ${ }^{2}, X$ Yi $^{1}$, Lijuan Xiong ${ }^{2}$, Yu Wang ${ }^{4}$ and Shijun $\mathrm{Li}^{3^{*}}$
}

\begin{abstract}
Background: Staphylococcus aureus (S. aureus), including methicillin-susceptible S. aureus (MSSA) and methicillinresistant S. aureus (MRSA), is an eminent human pathogen that can colonize the human host and cause severe lifethreatening infections. The development of a reliable, simple and rapid assay for detecting $\mathrm{S}$. aureus and identifying MRSA is important for diagnosis and follow-up treatment.

Methods: A novel molecular diagnosis technique, named multiplex loop-mediated isothermal amplification linked to a nanoparticle-based lateral flow biosensor (m-LAMP-LFB), was applied to detect all S. aureus species and identify MRSA. Two sets of primers were designed based on the femA gene (S. aureus-specific gene) and the mecA gene (encoding penicillin-binding protein 2a), and the multiple-LAMP products were analyzed using LFB. The m-LAMPLFB amplification conditions, including the target DNA concentration, reaction temperature and time, were optimized. The sensitivity and specificity of the m-LAMP-LFB method were tested in the current study, and the multiple-LAMP-LFB technology was applied to detect the MSSA and MRSA strains from clinical samples.

Results: The S. aureus- and MRSA-specific primers based on the femA and mecA genes allowed the multiple-LAMP technology to detect $S$. aureus and MRSA, respectively. The multiple-LAMP conditions were optimized at $63^{\circ} \mathrm{C}$ for $40 \mathrm{~min}$. The full process, including genomic DNA template preparation, LAMP, and product identification, could be achieved in $80 \mathrm{~min}$. The limit of detection (LoD) of the multiple-LAMP assay for femA and mecA detection was 100 fg of genomic DNA template per reaction. The specificity of m-LAMP-LFB detection was $100 \%$, and no crossreactions to non-S. aureus strains were observed.

(Continued on next page)
\end{abstract}

* Correspondence: zjumedjun@163.com

${ }^{3}$ Laboratory of Bacterial Infectious Disease of Experimental Center, Guizhou

Provincial Centre for Disease Control and Prevention, 73 Bageyan Road,

Guiyang, Guizhou 550004, P.R. China

Full list of author information is available at the end of the article

(c) The Author(s). 2020 Open Access This article is licensed under a Creative Commons Attribution 4.0 International License, which permits use, sharing, adaptation, distribution and reproduction in any medium or format, as long as you give appropriate credit to the original author(s) and the source, provide a link to the Creative Commons licence, and indicate if changes were made. The images or other third party material in this article are included in the article's Creative Commons licence, unless indicated otherwise in a credit line to the material. If material is not included in the article's Creative Commons licence and your intended use is not permitted by statutory regulation or exceeds the permitted use, you will need to obtain permission directly from the copyright holder. To view a copy of this licence, visit http://creativecommons.org/licenses/by/4.0/ The Creative Commons Public Domain Dedication waiver (http://creativecommons.org/publicdomain/zero/1.0/) applies to the data made available in this article, unless otherwise stated in a credit line to the data. 
(Continued from previous page)

Conclusion: The multiple-LAMP-LFB technique developed in the current study is a reliable, simple, rapid, specific and sensitive method to identify MSSA and MRSA infections for appropriate antibiotic therapy.

Keywords: Staphylococcus aureus, MRSA, Limit of detection, Loop-mediated isothermal amplification, Lateral flow biosensor

\section{Background}

Staphylococcus aureus, a gram-positive coccoid bacterium, is a common human pathogen that has the ability to cause a wide array of severe hospital and communityacquired infections, such as pneumonia, bacteremia, sepsis and toxic shock syndrome [1-3]. S. aureus infections have been typically treated with methicillin (a semisynthetic antibiotic), which was developed and applied to clinical practice in the late 1950s [4]. Unfortunately, a methicillin-resistant $S$. aureus (MRSA) strain was found among clinical isolates from patients hospitalized in 1960. By the 1980s, MRSA strains were globally epidemic in both community and healthcare settings [5-7]. According to a 2014 World Health Organization (WHO) report, MRSA was listed as one of the seven pathogens of international concern and has been associated with a high number of mortality and mortality $[8$, 9]. Thus, developing a reliable and rapid method of detection for the accurate differentiation of methicillinsusceptible $S$. aureus (MSSA) and MRSA isolates is necessary for the follow-up treatment and management of patients.

The traditional methods for the detection of MSSA and MRSA is based on cultivation [10] and include colony morphology, biochemical measurements and microdilution drug resistance testing; however, these methods are time-consuming and laborious. These methods require more than 2 days to identify MSSA or MRSA isolates. For this reason, the optimal treatment period is missed for many patients, or antibiotics are abused, resulting in multidrug resistance [11-13]. In recent decades, many molecular methods, such as polymerase chain reaction (PCR), multiplex PCR and real-time PCR, have been applied for detecting MSSA and MRSA isolates $[10,14,15]$. However, PCR-based methods require special experimental instruments and skilled personnel that may not be readily available in many resource-poor settings. Therefore, a cost-effective, simple, reliable, rapid, sensitive, and specific assay for the identification of MSSA and MRSA should be developed to improve treatment and prevent the spread and outbreak of these infections.

To overcome the drawbacks of PCR-based detection, a wide variety of isothermal amplification-based methods have been developed for use in molecular identification. Loop-mediated isothermal amplification (LAMP), as a reliable, low-cost, sensitive and rapid assay, has been widely applied to detect many bacterial pathogens, including Streptococcus pneumoniae, Salmonella and Brucella [16-18]. Unfortunately, the use of the multiplex LAMP (m-LAMP) method to distinguish MRSA from MSSA species has not been reported thus far. LAMP products have been analyzed by various methods, including agarose gel electrophoresis, visual inspection of color changes and turbidimetry changes [19-22]. However, these detection techniques are not specific for target genes and are likely to cause false positive results. To overcome this defect, a target-specific, visual and simple nanoparticle-based lateral flow biosensor (LFB) detection method was successfully designed and applied to analyze m-LAMP products in the current study. Hence, the multiplex-LAMP technique linked to a LFB detector (m-LAMP-LFB) was developed for the reliable, simple, specific, sensitive and visual identification of MSSA and MRSA strains by targeting the femA and mecA genes, respectively. femA is a $S$. aureusspecific gene that appears to be uniquely present in $S$. aureus as it shows no homology with other microbial genomes at GenBank by BLAST searches. $m e c A$ is an MRSA-specific gene that encodes penicillin-binding protein $2 \mathrm{a}$. The optimal amplification conditions and feasibility of the m-LAMP-LFB assay were confirmed with pure cultures and clinical samples.

\section{Materials and methods Materials instruments}

Bacterial genomic DNA extraction kits (QIAamp DNA minikits; Qiagen, Hilden, Germany) were purchased from Qiagen (Beijing, China). Universal isothermal amplification kits, a colorimetric indicator (malachite green, MG), and biotin-14-dCTP were obtained from Bei-Jing HaiTaiZhengYuan. Co., Ltd. (Beijing, China). The LFB materials, including the backing card, sample pad, absorbent pad, conjugate pad and nitrocellulose membrane (NC), were purchased from Jie-Yi Biotechnology. Co., Ltd. (Shanghai, China). Anti-FITC (rabbit antifluorescein antibody) and biotin-BSA (biotinylated bovine serum albumin) were purchased from Abcam. Co., Ltd. (Shanghai, China). Dye (Crimson red) streptavidincoated polymer nanoparticles $\left(129 \mathrm{~nm}, 10 \mathrm{mg} \mathrm{ml}^{-1} ; 100\right.$ $\mathrm{mM}$ borate, $\mathrm{pH} 8.5$, with $0.1 \% \mathrm{BSA}, 0.05 \%$ Tween 20 and $10 \mathrm{mM}$ EDTA) were purchased from Bangs Laboratories, Inc. (Indiana, USA). 


\section{Design of LAMP detection primers}

Based on the reaction mechanism of LAMP, two sets of specific primers were designed according to the target genes femA (GenBank Accession No. NC 007795) and mecA (GenBank Accession No. X52593) to detect S. aureus and MRSA, respectively. The primers were designed with Primer Explorer V4 (http://primerexplorer.jp/e/; Eiken Chemical Co., Ltd., Tokyo, Japan) online primer design software and checked with the basic local alignment search tool (BLAST). The primer positions are shown in Fig. 1, and the primer sequences and modifications are shown in Table 1. All of the primers were synthesized by TsingKe Biotech Co., Ltd. (Beijing, China) with HPLC purification grade.

Bacterial strains and genomic DNA template preparation In the current study, a total of 49 strains, including the $S$. aureus reference strain (ATCC 25923), 11 isolated MSSA strains, the methicillin resistant $S$. aureus reference strain (ATCC 43300), 16 MRSA isolated strains, and 20 non-S. aureus strains, were used for m-LAMPLFB detection (Table 2). Genomic DNA templates were obtained using DNA extraction kits in accordance with the manufacturer's instructions, and the concentration and purity were identified with a Nano drop ND-2000 (Beijing, China) at A260/280. The DNA templates were stored at $-20^{\circ} \mathrm{C}$ before use. The genomic DNA of $S$. aureus (ATCC 25923) and MRSA (ATCC 43300) were serially diluted to concentrations ranging from $10 \mathrm{ng} / \mu \mathrm{L}$ to $100 \mathrm{ag} / \mu \mathrm{L}(10 \mathrm{ng} / \mu \mathrm{L}, 10 \mathrm{pg} / \mu \mathrm{L}, 1 \mathrm{pg} / \mu \mathrm{L}, 100 \mathrm{fg} / \mu \mathrm{L}, 10$ $\mathrm{fg} / \mu \mathrm{L}, 1 \mathrm{fg} / \mu \mathrm{L}$, and $100 \mathrm{ag} / \mu \mathrm{L}$ ), which were used to optimize the reaction temperature, reaction time, and sensitivity.

\section{Gold nanoparticle-based lateral flow biosensor preparation}

The LFB platform was prepared according to a previous report [23]. Briefly, the LFB contained four components: an absorbent pad, NC membrane, sample pad, and conjugate pad. The components were assembled orderly on

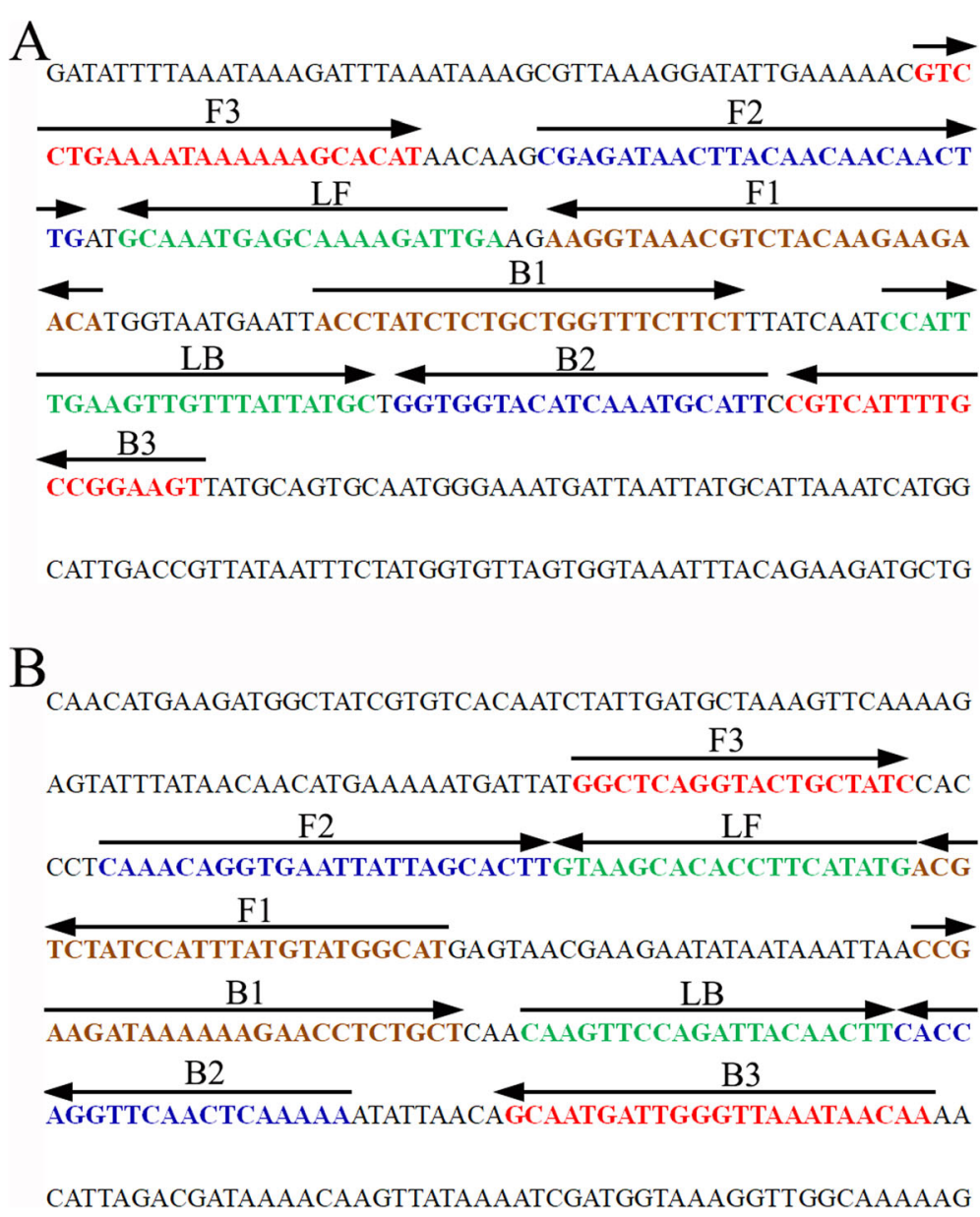

Fig. 1 Sequence and location of the femA (a) and $\operatorname{mec} A(\mathbf{b})$ genes used to design $m$-LAMP primers. The nucleotide sequences of the sense strand of the femA and mecA genes are shown in the diagram. Right arrows and left arrows indicate sense and complementary sequences, respectively, which were used in the current study 
Table 1 The primers used in the present study

\begin{tabular}{|c|c|c|c|}
\hline Primer name & Sequence and modifications & Length & Gene \\
\hline F3 & 5'-GTCCTGAAAATAAAAAAGCACAT-3' & $23 \mathrm{nt}$ & femA \\
\hline B3 & 5'-ACTTCCGGCAAAATGACG-3' & $18 \mathrm{nt}$ & \\
\hline FIP & 5'-TGTTCTTCTTGTAGACGTTTACCTT-CGAGATAACTTACAACAACAACTTG-3' & 50 mer & \\
\hline FIP* & 5'-Dig- TGTTCTTCTTGTAGACGTITACCTT-CGAGATAACTTACAACAACAACTTG -3' & 50 mer & \\
\hline BIP & 5'-ACCTATCTCTGCTGGTTTCTTCT-AATGCATTTGATGTACCACC-3' & $43 \mathrm{nt}$ & \\
\hline LF & 5'-TCAATCTTTTGCTCATTTGC-3' & $20 \mathrm{nt}$ & \\
\hline $\mathrm{LF}^{*}$ & 5'-Biotin-TCAATCTITTGCTCATTTGC-3' & $20 \mathrm{nt}$ & \\
\hline LB & 5'-CCATTTGAAGTTGTTTATTATGC-3' & $23 \mathrm{nt}$ & \\
\hline F3 & 5'-GGCTCAGGTACTGCTATC-3' & $18 \mathrm{nt}$ & mecA \\
\hline B3 & 5'-TTGTTATTTAACCCAATCATTGC-3' & $23 \mathrm{nt}$ & \\
\hline FIP & 5'-ATGCCATACATAAATGGATAGACGT-CAAACAGGTGAATTATTAGCACTT-3' & $49 \mathrm{nt}$ & \\
\hline FIP* & 5'-FAM-ATGCCATACATAAATGGATAGACGT-CAAACAGGTGAATTATTAGCACTT-3' & $49 \mathrm{nt}$ & \\
\hline BIP & 5'-CCGAAGATAAAAAAGAACCTCTGCT-TITTGAGTTGAACCTGGTG-3' & $45 \mathrm{nt}$ & \\
\hline LF & 5'-CATATGAAGGTGTGCTTAC-3' & $19 \mathrm{nt}$ & \\
\hline$L^{*}$ & 5'-Biotin-CATATGAAGGTGTGCTTAC-3' & $19 \mathrm{nt}$ & \\
\hline LB & 5'-CAAGTTCCAGATTACAACTT-3' & $20 \mathrm{nt}$ & \\
\hline
\end{tabular}

Note: femA-FIP*, 5'-labeled with Dig when used in LAMP-LFB assay; femA-LF*, 5'-labeled with biotin when used in LAMP-LFB assay mecA-FIP*, 5'-labeled with FAM when used in the LAMP-LFB assay; mecA-LF*, 5'-labeled with biotin when used in the LAMP-LFB assay;

Abbreviations: Dig digoxigenin, FAM carboxyfluorescein, $n t$ nucleotide, mer monomeric unit

a backing card. The capture reagents, including anti-Dig, anti-FAM, and biotin-BSA, were immobilized by physical adsorption on the reaction regions. Then, anti-Dig was immobilized at test line 1 (TL1) (MSSA), and antiFAM was immobilized at test line 2 (TL2) (MRSA), while biotin-BSA was immobilized at the control line (CL); each line was separated by $5 \mathrm{~mm}$. SA-PNPs (dye streptavidin-coated polymer nanoparticles) were gathered on the conjugate pad. The prepared biosensors were preserved in a plastic box with a desiccant gel at room temperature before use.

\section{The standard LAMP reaction}

The single LAMP reactions for MSSA or MRSA were performed in $25 \mu \mathrm{l}$ reaction systems as previously described [18]. Briefly, 0.4 $\mu \mathrm{M}$ each outer primer, F3 and B3, $0.8 \mu \mathrm{M}$ each loop primer, $\mathrm{LF}^{*}$ and $\mathrm{LB}, 1.6 \mu \mathrm{M}$ each inner primer, FIP* and BIP, $0.4 \mathrm{mM}$ biotin-14-dCTP, $1 \mu \mathrm{l}(8 \mathrm{U})$ of Bst DNA polymerase, $12.5 \mu \mathrm{l}$ of $2 \times$ reaction buffer, and $1 \mu \mathrm{l}$ of DNA template were added to a tube. The mixtures were heated at $64{ }^{\circ} \mathrm{C}$ for $1 \mathrm{~h}$. Genomic DNA from non-S. aureus strains, including Pseudomonas aeruginosa and Enterococcus faecalis, was used as a negative control (NC), and double distilled water (DW) was used as the template in the blank control (BC).

The m-LAMP reaction was performed in a one-step reaction in a $25 \mu \mathrm{l}$ reaction system containing $12.5 \mu \mathrm{l}$ of $2 \times$ reaction buffer; $0.2 \mu \mathrm{M}$ each outer primer, femA-F3, femA-B3, mecA-F3 and mecA-B3; $0.4 \mu \mathrm{M}$ each loop primer, femA-LF*, femA-LB, mecA-LF* and mecA-LB;
$0.8 \mu \mathrm{M}$ each inner primer, femA-FIP*, femA-BIP, mecAFIP* and mecA-BIP; $0.4 \mathrm{mM}$ biotin-14-dCTP; $1 \mu \mathrm{l}(8 \mathrm{U})$ of Bst DNA polymerase; and $1 \mu \mathrm{l}$ of DNA template. The reaction conditions were as described above.

\section{Detection of LAMP products}

Colorimetric indicator (malachite green, MG) and lateral flow biosensor (LFB) methods were applied for the determination and verification of the femA-LAMP, mecALAMP, and m-LAMP products. For the products amplified effectively, the color changed from colorless to light green in the MG assay. However, the color of the negative and blank controls remained colorless. The strategy of visualization of LAMP products with LFB was as previously described [24].

\section{Temperature optimization of the LAMP assays}

The effect of temperature on each LAMP reaction (femA-LAMP and mecA-LAMP) was tested during the amplification stage. Reaction temperatures ranging from 60 to $67^{\circ} \mathrm{C}$ with $1{ }^{\circ} \mathrm{C}$ intervals were tested. Reaction mixtures with $1 \mu \mathrm{L}$ of genomic DNA from $P$. aeruginosa and $E$. faecalis were used as negative controls (NCs), and $1 \mu \mathrm{L}$ of distilled water (DW) was used as a blank control. The LAMP amplicons were analyzed by examining the turbidity of the products. The curves of the DNA concentrations of each amplified product are shown in the graph. Turbidity $>0.1$ was considered positive. 
Table 2 Bacterial strains used in the current study

\begin{tabular}{|c|c|c|c|c|c|}
\hline \multirow[t]{2}{*}{ No. } & \multirow[t]{2}{*}{ Bacteria } & \multirow[t]{2}{*}{ Strain no. (source of strains) } & \multirow{2}{*}{$\begin{array}{l}\text { No. of } \\
\text { strains }\end{array}$} & \multicolumn{2}{|c|}{ m-LAMP-LFB result ${ }^{b}$} \\
\hline & & & & femA & mecA \\
\hline 1 & S. aureus (MSSA) & ATCC 25923 & 1 & $P$ & N \\
\hline 2 & S. aureus (MSSA) & Isolated strains (2nd GZUTCM) & 11 & $P$ & $\mathrm{~N}$ \\
\hline 3 & S. aureus (MRSA) & ATCC 43300 & 1 & P & P \\
\hline 4 & S. aureus (MRSA) & Isolated strains (2nd GZUTCM) & 17 & $P$ & $P$ \\
\hline 5 & Enterococcus faecalis & ATCC 29212 & 1 & N & N \\
\hline 6 & Pseudomonas aeruginosa & ATCC 27853 & 1 & N & N \\
\hline 7 & Shigella flexneri & Isolated strains (2nd GZUTCM) & 1 & N & N \\
\hline 8 & Listeria monocytogenes & Isolated strains (2nd GZUTCM) & 1 & N & N \\
\hline 9 & Mycobacterium tuberculosis & Isolated strains (GZCDC) & 1 & N & N \\
\hline 10 & Acinetobacter baumannii & Isolated strains (2nd GZUTCM) & 1 & N & N \\
\hline 11 & Bacillus cereus & Isolated strains (GZCDC) & 1 & N & $\mathrm{N}$ \\
\hline 12 & Vibrio parahaemolyticus & Isolated strains (GZCDC) & 1 & N & N \\
\hline 13 & Leptospira interrogans & Isolated strains (GZCDC) & 1 & N & N \\
\hline 14 & Enterohemorrhagic Escherichia coli & Isolated strains (2nd GZUTCM) & 1 & N & N \\
\hline 15 & Enteroaggregative Escherichia coli & Isolated strains (2nd GZUTCM) & 1 & N & N \\
\hline 16 & Streptococcus pneumoniae & Isolated strains (2nd GZUTCM) & 1 & N & N \\
\hline 17 & Staphylococcus saprophyticus & Isolated strains (GZCDC) & 1 & N & $\mathrm{N}$ \\
\hline 18 & Enterotoxigenic Escherichia coli & Isolated strains (2nd GZUTCM) & 1 & N & N \\
\hline 19 & Invasive Escherichia coli & Isolated strains (2nd GZUTCM) & 1 & N & N \\
\hline 20 & Haemophilus parainfluenzae & Isolated strains (2nd GZUTCM) & 1 & N & N \\
\hline 21 & Shigella boydii & Isolated strains (2nd GZUTCM) & 1 & N & N \\
\hline 22 & Enteropathogenic Escherichia coli & Isolated strains (GZCDC) & 1 & N & N \\
\hline 23 & Klebsiella pneumoniae & Isolated strains (2nd GZUTCM) & 1 & N & N \\
\hline 24 & Bordetella parapertussis & Isolated strains (GZCDC) & 1 & N & $\mathrm{N}$ \\
\hline
\end{tabular}

${ }^{a}$ ATCC American Type Culture Collection, 2nd GZUTCM Second Affiliated Hospital, Guizhou University of Traditional Chinese Medicine, GZCDC Guizhou Provincial Center for Disease Control and Prevention

bP Positive, $N$ Negative

\section{Analytical sensitivity of LAMP-LFB assays}

The sensitivity of each LAMP-LFB reaction (femALAMP-LFB, mecA-LAMP-LFB, and m-LAMP-LFB) was determined using $1 \mu \mathrm{l}$ of the serial dilution (10 ng, $10 \mathrm{pg}$, $1 \mathrm{pg}, 100 \mathrm{fg}, 10 \mathrm{fg}, 1 \mathrm{fg}$ and $100 \mathrm{ag}$ per microliter) of the extracted genomic DNA. The LAMP-LFB reactions were carried out as described above, and the results were tested using a colorimetric indicator (MG) and LFB. The limit of detection (LoD) of single and multiplex reactions was verified as the last dilution of each positive test. Three replicates were tested for each dilution.

\section{Optimization of the amplification time for the multiplex- LAMP-LFB assay}

To optimize the reaction time of $\mathrm{m}$-LAMP-LFB, four amplification times (20, 30, 40 and $50 \mathrm{~min}$ ) were evaluated. The LAMP-LFB reactions were carried out as described above, and the results were tested using LFB. Each amplification time was tested at least three times.

\section{Specificity analysis of m-LAMP-LFB detection}

To determine the specificity of the m-LAMP-LFB assay, genomic DNA (at least $10 \mathrm{ng}$ per microliter) from 12 MSSA strains, 17 MRSA strains, and 20 non-S. aureus strains (Table 2) was used for m-LAMP, and all of the results were tested using the LFB method. All examinations were confirmed at least three times.

\section{Application of the m-LAMP-LFB method to analyze the clinical samples}

To verify the applicability of the m-LAMP-LFB method for detecting $S$. aureus and identifying MRSA strains, a total of 63 whole blood samples, which were suspected of $S$. aureus infection, were collected from the Second Affiliated Hospital of Guizhou University of Traditional Chinese Medicine. The clinical samples were detected for MSSA and MRSA using traditional culture, PCR, and m-LAMP-LFB methods. Traditional culture methods included blood culture, colony morphology, Gram 
staining, biochemical identification, and methicillin susceptibility testing. PCR diagnosis was carried out using $S$. aureus- and MRSA-specific primers targeting the fem $A$ and $m e c A$ genes, respectively. The m-LAMP-LFB detection was performed as described above.

\section{Results \\ Verification and analysis of femA- and mecA-LAMP products}

To confirm the amplification with the two sets of LAMP primers, the femA-, mecA-, and m-LAMP mixtures were incubated at a constant temperature of $63{ }^{\circ} \mathrm{C}$ for $1 \mathrm{~h}$. Then, the femA-, mecA-, and m-LAMP products were analyzed with colorimetric indicator (MG) and lateral flow biosensor (LFB) methods, respectively. The color of the positive results in the femA-, mecA-, and m-LAMP reactions changed from colorlessness to bright green, while the negative and blank control reactions remained colorless (Fig. 2a, c). LFB was used for further confirmation of $\mathrm{femA}$-, $m e c A-$, and m-LAMP. For femA-LAMP detection, two crimson red bands (CL and TL1) appeared, indicating positive results, and $\mathrm{CL}$ and TL2 were visible for $m e c A$-LAMP, indicating successful amplification, while the negative and blank controls only appeared as a crimson red line (CL) in the biosensor (Fig. 2b, d). Therefore, the results suggested that the two sets of LAMP primers for $f e m A$ and $m e c A$ detection were valid for the development of the m-LAMP techniques.

\section{Optimal amplification temperature for femA- and mecA- LAMP}

The reaction temperature is crucial for LAMP. In this study, the reaction temperature of femA- and mecALAMP was tested at different temperatures (from 60 to $67^{\circ} \mathrm{C}$ with $1{ }^{\circ} \mathrm{C}$ intervals) with genomic template $(10 \mathrm{pg} /$ $\mu \mathrm{l})$ extracted from purified cultures (ATCC 43300). The LAMP protocol was performed as described above, and the $f e m A$ - and mecA-LAMP reactions were monitored by means of real-time turbidity measurements. Kinetic graphs were recorded at all temperatures. The results showed that femA-LAMP was amplified faster in the temperature range from 63 to $66^{\circ} \mathrm{C}$, and mecA-LAMP was amplified faster at 61 to $67^{\circ} \mathrm{C}$ (Fig. 3). Hence, the amplification temperature of $63^{\circ} \mathrm{C}$ was considered the optimal temperature for the rest of the m-LAMP reactions in the present study.

\section{Sensitivity of femA- and mecA-LAMP detection}

The sensitivity of femA- and mecA-LAMP detection was evaluated with serially diluted genomic DNA at concentrations ranging from $10 \mathrm{ng}$ to $100 \mathrm{ag}$ per microliter. The LAMP amplicons were analyzed by visual inspection with MG reagents and lateral flow biosensors. The CL and TL1 lines appeared on the biosensor, showing positive results for the femALAMP assay, and two crimson lines (CL and TL2) were observed on the biosensor, indicating positive results for mecA-LAMP detection. For the negative
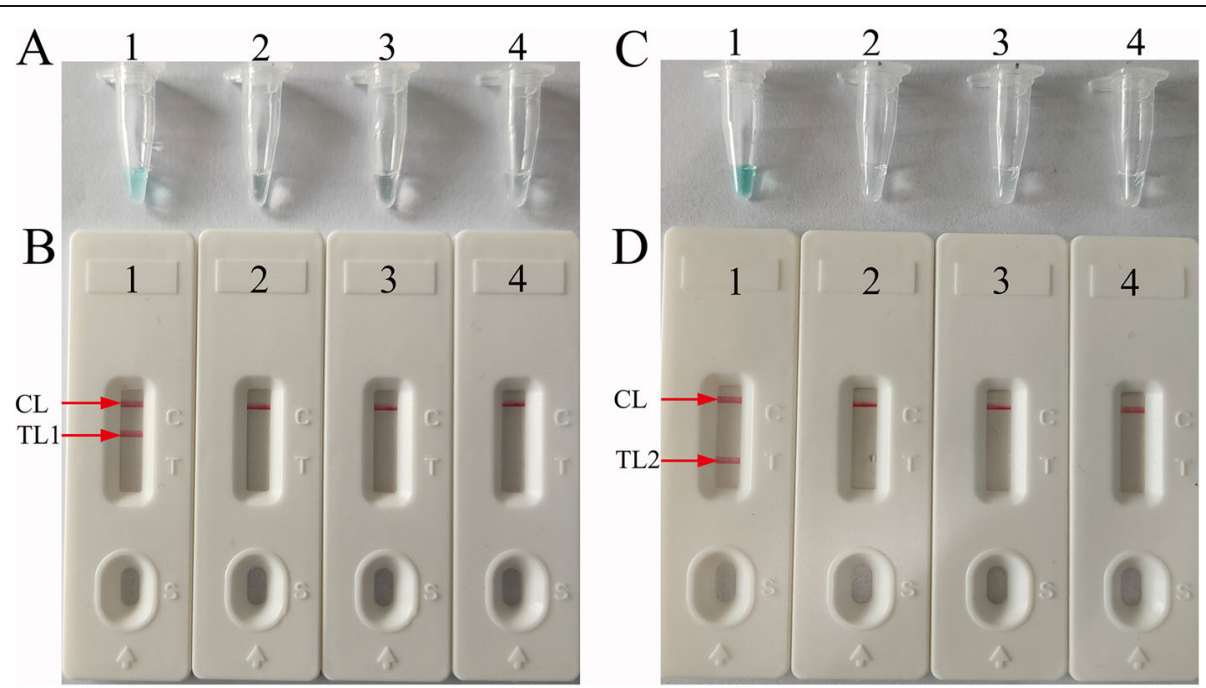

Fig. 2 Detection and verification of femA-and mecA-LAMP products. a, b Color and lateral flow biosensor detection of femA-LAMP products. Tube A1/Biosensor B1, positive amplification of the femA gene (S. aureus, ATCC25923); Tube A2 /Biosensor B2, negative amplification (Pseudomonas aeruginosa); Tube A3/Biosensor B3, negative amplification (Enterococcus faecalis); Tube 4/Biosensor 4, blank control (DW). c, d Color and lateral flow biosensor detection of mecA-LAMP products. Tube A1/Biosensor B1, positive amplification of the mecA gene (methicillin-resistant $S$. aureus, ATCC43300); Tube A2/Biosensor B2, negative amplification (Pseudomonas aeruginosa); Tube A3/Biosensor B3, negative amplification (Enterococcus faecalis); Tube 4/Biosensor 4, blank control (DW) 

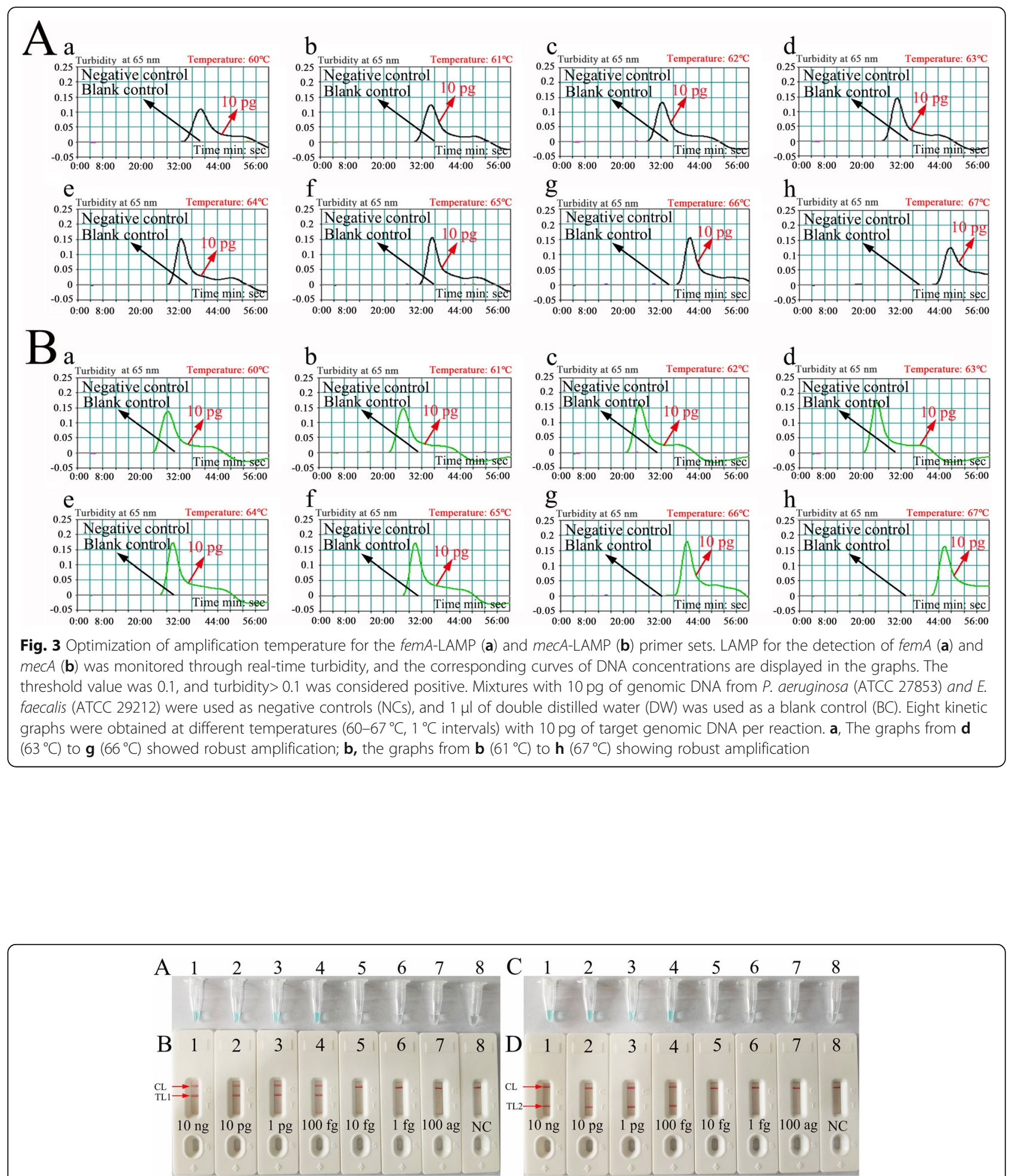

Fig. 4 Sensitivity analysis of femA-LAMP $(\mathbf{a}, \mathbf{b})$ and meCA-LAMP $(\mathbf{c}, \mathbf{d})$ detection with serial dilutions of genomic DNA extracted from MSSA and MRSA strains, respectivelyTwo detection methods involving a colorimetric indictor (MG; $\mathbf{a}, \mathbf{c})$ or lateral flow biosensor (b, $\mathbf{d}$ ) were used to analyze the amplification products. The genomic DNA was serially diluted (10 ng, 10 pg, 1 pg, $100 \mathrm{fg}, 10 \mathrm{fg}, 1 \mathrm{fg}$ and 100 ag per microliter) and subjected to standard LAMP. Tubes A1-A7 (Biosensors B1-B7), S. aureus (ATCC25923) genomic templates (10 ng-100 ag); Tube A8 (Biosensor B8), negative control (DW). The LoD of femA-LAMP detection was $100 \mathrm{fg}$ of genomic template per reaction. Tubes C1-C7 (Biosensors D1-D7), methicillinresistant S. aureus (ATCC43300) genomic templates (10 ng-100 ag); Tube C8 (Biosensor D8), blank control (DW). The LoD of mecA-LAMP detection was $100 \mathrm{fg}$ of genomic template per reaction. 


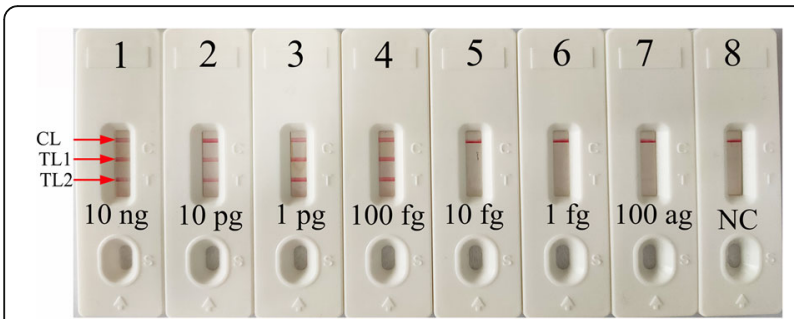

Fig. 5 Sensitivity analysis of m-LAMP detection with serial dilutions of genomic DNA extracted from the MRSA strain. Two sets of primers targeting the femA and mecA genes were simultaneously added to a reaction vessel, and the LOD of $M$-LAMP for detecting $S$. aureus and identifying MRSA was analyzed with a lateral flow biosensor. Biosensors 1-8 represent the genomic DNA (methicillinresistant S. aureus, ATCC43300) amounts of $10 \mathrm{ng}, 10 \mathrm{pg}, 1 \mathrm{pg}, 100 \mathrm{fg}$, $10 \mathrm{fg}, 1 \mathrm{fg}$ and $100 \mathrm{ag}$ per reaction and blank control (DW), respectively. The LoD of the m-LAMP assay for femA and mecA detection was $100 \mathrm{fg}$ of genomic template per reaction of DNA per reaction (Fig. 5), which was consistent with single LAMP detection (Figs. 4 and 5).

\section{Optimization of amplification time for m-LAMP-LFB detection}

To obtain an optimal reaction time for m-LAMP, four reaction times $(20,30,40$, and $50 \mathrm{~min})$ were tested at the optimal amplification temperature $\left(63^{\circ} \mathrm{C}\right)$. The results showed that the LoD of the genomic DNA template (100 fg of MRSA per reaction) was detected (displayed TL1, TL2 and CL) when the m-LAMP lasted $40 \mathrm{~min}$ (Fig. 6). Hence, a reaction time of $40 \mathrm{~min}$ was considered the optimal reaction time for m-LAMP detection. In summary, the whole detection procedure, including target genomic DNA preparation (30 min), m-LAMP (40 $\mathrm{min}$ ) and analysis of results $(2 \mathrm{~min})$, could be completed within 80 min.

controls, only the CL line appeared on the biosensor, indicating negative results. The results showed that the LoD of mecA-LAMP was $100 \mathrm{fg}$ per reaction, which was the same as the LoD of the femA-LAMP assay (Fig. 4).

\section{Sensitivity of $m$-LAMP detection}

After m-LAMP was performed, the products were directly analyzed using LFB. The CL, TL1, and TL2 bands became crimson on the biosensor, reporting positive results for the femA and mecA genes. Only the CL line appeared on the biosensor, indicating negative results. The results showed that the LoD of m-LAMP-LFB for simultaneously assaying $\mathrm{fem} A$ and $m e c A$ genes was also $100 \mathrm{fg}$

\section{Specificity of the m-LAMP assay}

The specificity of m-LAMP detection was confirmed with MSSA, MRSA, and non-S. aureus isolates (Table 2). The genomic DNA extracted from MSSA and MRSA strains presented positive results. Three crimson lines (TL1, TL2 and CL) were displayed on the LFB, indicating positive results for the MRSA isolates. TL1 and CL appeared on the LFB, indicating positive results for the MSSA isolates. Other non-S. aureus strains and the blank control showed negative results (Fig. 7). Hence, the results confirmed that the m-LAMP-LFB method could accurately identify $S$. aureus and differentiate MRSA from all $S$. aureus strains.
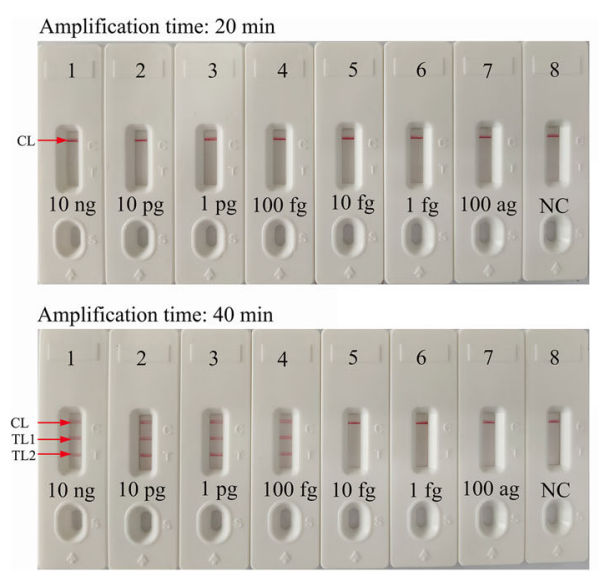
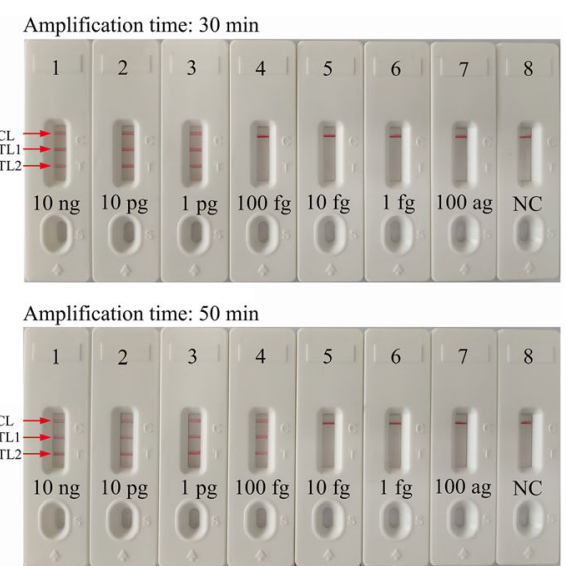

Fig. 6 Optimization of the amplification time for m-LAMP detection. Different amplification times (a, 20 min; b, 30 min; $\mathbf{c}$, 40 min; d, 50 min) were tested at $63^{\circ} \mathrm{C}$. Biosensors 1, 2, 3, 4, 5, 6, 7, and 8 represent genomic DNA (methicillin-resistant S. aureus, ATCC43300) levels of $10 \mathrm{ng}, 10 \mathrm{pg}, 1 \mathrm{pg}$, $100 \mathrm{fg}, 10 \mathrm{fg}, 1 \mathrm{fg}$ and $100 \mathrm{ag}$ target template per reaction and negative control (DW), respectively. The best sensitivity was observed when the amplification lasted for $40 \mathrm{~min}$ (c) 


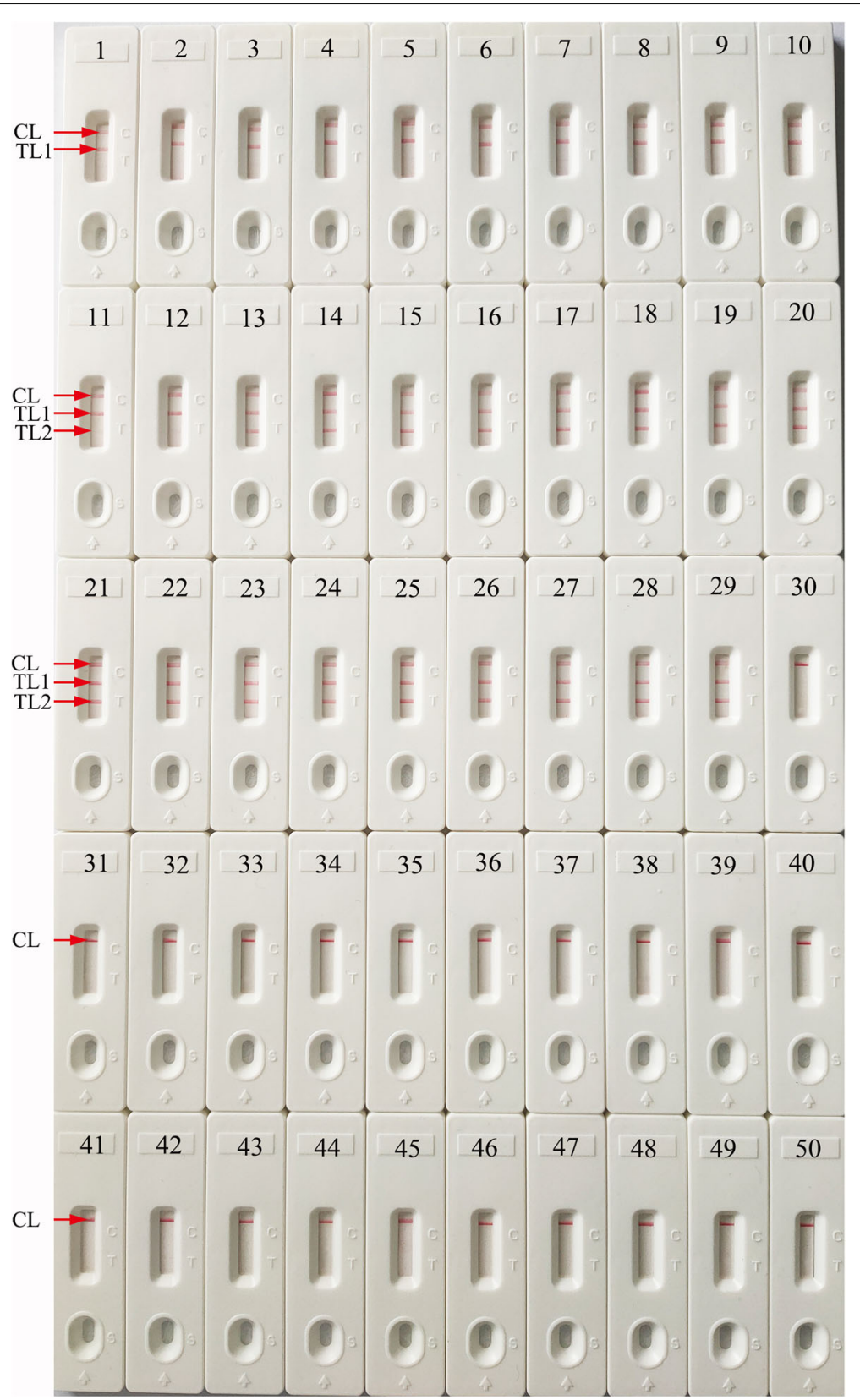

Fig. 7 Specificity analysis of m-LAMP-LFB detection using different strains. The m-LAMP reactions were carried out using different genomic DNA as templates, and each of the amplification products was determined by means of the visual LFB method. Biosensor 1, MSSA (ATCC 25923); Biosensors 2-12; eleven isolated strains of MSSA from the Second Affiliated Hospital, Guizhou University of Traditional Chinese Medicine; Biosensor 13, MRSA (ATCC 43300); Biosensors 14-29, sixteen isolated strains of MRSA from the Second Affiliated Hospital, Guizhou University of Traditional Chinese Medicine; Biosensors 30-49, Enterococcus faecalis, Pseudomonas aeruginosa, Shigella Flexneri, Listeria monocytogenes,

Mycobacterium tuberculosis, Acinetobacter baumannii, Bacillus cereus, Vibrio parahaemolyticus, Leptospira interrogans, enterohemorrhagic Escherichia coli, enteroaggregative Escherichia coli, Streptococcus pneumonia, Staphylococcus saprophyticus, enterotoxigenic Escherichia coli, invasive Escherichia coli, Hemophilus parainfluenza, Shigella boydii, enteropathogenic Escherichia coli, Klebsiella pneumonia, Bordetella parapertussis, respectively; biosensor 50, blank control (DW)

Feasibility of the m-LAMP-LFB method using whole blood samples

To further demonstrate the feasibility of m-LAMP-LFB as a valuable method for the detection of MRSA and MRSA, 63 whole blood samples of suspected S. aureus- infected patients were collected from the Second Affiliated Hospital of Guizhou University of Traditional Chinese Medicine and tested by conventional culturebiotechnical methods, PCR detection, and m-LAMP-LFB assays. The results showed that 16 of 63 samples had 
Table 3 Comparison of conventional culture, PCR and m-LAMPLFB methods to identify MSSA and MRSA in clinical samples

\begin{tabular}{llll}
\hline Detection & \multicolumn{3}{l}{ Clinical samples $(n=63)$} \\
\cline { 2 - 4 } method & MSSA-Positive & MRSA-Positive & Negative \\
\hline Culture & 16 & 12 & 35 \\
PCR & 14 & 9 & 40 \\
m-LAMP-FB & 16 & 12 & 35 \\
\hline
\end{tabular}

been verified as MSSA-positive, and 12 of 63 samples had been verified as MRSA-positive through traditional culture techniques. The m-LAMP-LFB assay results were consistent with the traditional cultivation detection results. However, using PCR, only 14 and 9 samples were confirmed as MSSA- and MRSA-positive results, respectively (Table 3 ). These results suggested that the m-LAMP-LFB assay established in the current study could be used as an advanced tool to detect all $S$. aureus strains and separate MRSA from MSSA.

\section{Discussion}

S. aureus, a 'Janus-faced' bacterium, is a commensal species and a pathogenic microorganism [25-27]. It is estimated that $20-30 \%$ of adult populations carry $S$. aureus in their nares; although this bacterium is commonly found, it only causes invasive infection when the host immune system is weakened [28-30]. In nosocomial settings, $S$. aureus is widely distributed in the environment, on surgical instrument surfaces, and on implanted medical devices, including prosthetic joints, catheters, and artificial heart valves $[11,31]$. After S. aureus invades the human body, the pathogen rapidly enters the bloodstream and then diffuses into vital organs, causing pathological injury, such as osteomyelitis, endocarditis, and descending urinary tract infections [28]. Importantly, since methicillin-resistant $S$. aureus has emerged and spread worldwide, $S$. aureus has disrupted both the healthcare setting and community [4,30], resulting in a huge socioeconomic burden in both developed and developing areas. The detection and identification of MSSA and MRSA is essential in cases of suspected $S$. aureus infections. However, traditional methods, including culture-based techniques, colony morphology, Gram staining, biochemical identification, methicillin susceptibility testing, and PCR-based detection (traditional PCR, multiple PCR and real-time PCR approaches), are time consuming and require expensive instruments. Moreover, the accurate interpretation of the results requires trained experts [14, 32]. Herein, developing a reliable, rapid, low-cost, simple, specific and sensitive detection method to accurately differentiate MSSA and MRSA is essential for disease diagnosis and therapy.

In the current study, m-LAMP-LFB detection targeting the $f e m A$ and $m e c A$ genes was successfully established to assay all $S$. aureus species and identify MRSA. The sequences of $S$. aureus-LAMP primers were designed using the $f e m A$ gene, which appears to be a unique feature of S. aureus and is not found in other Staphylococcus species [10]. Moreover, the MRSA-LAMP primers were designed with the mecA gene, encoding the lowaffinity penicillin-binding protein $2 \mathrm{a}$ (PBP2a), which is associated with the methicillin resistance of $S$. aureus species [33]. The specificity of the m-LAMP assay was confirmed with genomic DNA from 12 MSSA, 17 MRSA, and 20 non-S. aureus isolates. m-LAMP detection of the femA gene identified S. aureus with 100\% specificity, and the mecA gene identified MRSA with 100\% specificity (Fig. 7).

In the present study, a nanoparticle-based lateral flow biosensor (LFB) was applied to analyze LAMP products. Although the amplification results could be detected equally with the turbidity and MG methods used in the current study, the LFB was deemed the preferred method for analyzing LAMP products; the LFB provides more visualization and does not require special instruments, regents and processes [34, 35]. In particular, the LFB applied in this study can simultaneously and visually detect two target genes ( $f e m A$ and $m e c A)$ in a single test. Compared with conventional culture and PCR-based methods, the m-LAMP-LFB technique is more sensitive, time-saving and cost-saving. The newly developed $\mathrm{m}$ LAMP-LFB method was able to detect $100 \mathrm{fg}$ of genomic DNA (Figs. 4 and 5). The entire detection process, including template preparation (approximately $30 \mathrm{~min}$ ), isothermal amplification (40 min) and LFB reading (approximately $2 \mathrm{~min}$ ), could be accomplished within 80 min. The total cost of one test, including genomic DNA extraction (approximately \$1 USD), LAMP reaction (approximately \$3.5 USD) and LFB reading (approximately $\$ 2$ USD), is estimated to be $\$ 6.5$ USD, which is cheaper than normal PCR-based methods. In addition, the advanced technique can decrease labor costs because performing the LAMP-LFB assay does not require skilled technical personnel. In conclusion, the newly developed $\mathrm{m}$-LAMP-LFB technique in this study is a rapid, reliable and low-cost assay for the identification of MSSA and MRSA, and this technique can save detection time and help determine the optimal treatments for patients in a timely manner. In addition, accurate, timely and lowcost testing can reduce the patient's financial burden, especially in resource-constrained regions of the world.

\section{Conclusions}

In the current study, a reliable, rapid and simple mLAMP-LFB technique based on the $f e m A$ and $m e c A$ genes was successfully developed for assaying $S$. aureus and identifying MRSA. This method could reliably, specifically, sensitively and rapidly detect all $S$. aureus species and 
identify MRSA isolates in samples. The amplification products were analyzed with LFB, which was objective, rapid, and easily interpretable. Hence, the m-LAMP-LFB assay could be considered a useful method for the reliable and rapid detection of $S$. aureus and identification of MRSA in clinical samples, particularly in resourceconstrained regions of the world.

\section{Abbreviations \\ MSSA: Methicillin susceptible Staphylococcus aureus; MRSA: Methicillin resistant Staphylococcus aureus; LAMP: Loop-mediated isothermal amplification; LFB: Lateral flow biosensor; m-LAMP-LFB: multiplex loop- mediated isothermal amplification linked to a nanoparticle-based lateral flow biosensor; LOD: Limit of detection; WHO: WORLD Health Organization; PCR: Polymerase chain reaction; MG: Malachite green; ATCC: American type culture collection; 2nd GZUTCM: Second Affiliated Hospital, Guizhou University of Traditional Chinese Medicine; GZCDC: Guizhou Provincial Center for Disease Control and Prevention; Dig: Digoxigenin; \\ FAM: Carboxyfluorescein; nt: nucleotide; mer: monomeric unit: TL1: Test line 1; TL2: Test line 2; CL: Control line; NC: Negative control; BC: Blank control; DW: Distilled water; PBP2a: Penicillin-binding protein 2a}

\section{Acknowledgments}

We thank all the medical workers in both the 2nd GZUTCM and GZCDC for their cooperation in this study.

\section{Authors' contributions}

$X C, K M$, and SL conceived and designed the study. XC and SL participated in primer design. $\mathrm{XC}, \mathrm{KM}, \mathrm{XY}, \mathrm{LX}$ and $\mathrm{YW}$ contributed to all the laboratory work. $\mathrm{KM}$, and $\mathrm{LX}$ contributed to the data collection. $\mathrm{XC}, \mathrm{XY}$ and SL performed the statistical analysis. $X C$ and SL wrote the initial draft of the manuscript. XC, YW, and SL revised the manuscript. All the authors read and approved the final manuscript.

\section{Funding}

This study was supported by the Program of the Scientific and Technological in Guiyang City (Grant No.Zhu Ke He [2020]1001), the High-Level Creative Talents Cultivation in Guizhou Province (Qian Ke He [2016]4021), the Program of Scientific and Technological Innovation Team of Guizhou Province (Grant No. Qian Ke He Platform talent [2018]5606), the Guizhou Provincial Special Funds for Science and Technology Supporting Program ([2019]1186, [2018]5767), and the Program of Science and Technology of Guizhou Provincial Health Commission (gzwjkj2018-1-012).

\section{Availability of data and materials}

The datasets used and/or analyzed during the current study are available from the corresponding author on reasonable request.

\section{Ethics approval and consent to participate}

The study was approved by the Human Ethics Committee of the Second Affiliated Hospital of Guizhou University of Traditional Chinese Medicine and complied with the Declaration of Helsinki. All data/isolates were analyzed anonymously.

\section{Consent for publication}

Not applicable.

\section{Competing interests}

All of the authors declare that there are no competing interests in this article.

\section{Author details}

${ }^{1}$ Central Laboratory of the Second Affiliated Hospital, Guizhou University of Traditional Chinese Medicine, Guiyang, Guizhou 550003, P.R. China. ${ }^{2}$ The Second Clinical College, Guizhou University of Traditional Chinese Medicine, Guiyang, Guizhou 550003, P.R. China. ${ }^{3}$ Laboratory of Bacterial Infectious Disease of Experimental Center, Guizhou Provincial Centre for Disease Control and Prevention, 73 Bageyan Road, Guiyang, Guizhou 550004, P.R.
China. ${ }^{4}$ Department of Clinical Laboratory Centre, The First People's Hospital of Guiyang, Guiyang, Guizhou 55004, P.R. China.

Received: 13 December 2019 Accepted: 9 July 2020

Published online: 17 July 2020

\section{References}

1. Turner NA, Sharma-Kuinkel BK, Maskarinec SA, Eichenberger EM, Shah PP, Carugati M, Holland TL, Fowler VJ. Methicillin-resistant Staphylococcus aureus: an overview of basic and clinical research. Nat Rev Microbiol. 2019; 17(4):203-18.

2. Carrel M, Perencevich EN, David MZ. USA300 methicillin-resistant Staphylococcus aureus, United States, 2000-2013. Emerg Infect Dis. 2015; 2(11):1973-80

3. Carroll DE, Marr I, Huang GKL, Holt DC, Tong SYC, Boutlis CS. Staphylococcus aureus prostatic abscess: a clinical case report and a review of the literature. BMC Infect Dis. 2017;17(1):509-7.

4. Peterson LR, Schora DM. Methicillin-resistant Staphylococcus aureus control in the 21st century: laboratory involvement affecting disease impact and economic benefit from large population studies. J Clin Microbiol. 2016; 54(11):2647-54.

5. Okwu MU, Olley M, Akpoka AO, Izevbuwa OE. Methicillin-resistant Staphylococcus aureus (MRSA) and anti-MRSA activities of extracts of some medicinal plants: a brief review. AIMS Microbiolol. 2019:5(2):117.

6. Naimi HM, Rasekh H, Noori AZ, Bahaduri MA. Determination of antimicrobial susceptibility patterns in Staphylococcus aureus strains recovered from patients at two main health facilities in Kabul, Afghanistan. BMC Infect Dis. 2017;17(1):737.

7. McCarthy H, Rudkin JK, Black NS, Gallagher L, O'Neill E, O'Gara JP. Methicillin resistance and the biofilm phenotype in Staphylococcus aureus. Front Cell Infect Mi. 2015;5:1.

8. Kong EF, Johnson JK, Jabra-Rizk MA. Community-associated methicillinresistant Staphylococcus aureus: an enemy amidst us. PLoS Pathog. 2016; 12(10):e1005837.

9. Che Hamzah AM, Yeo CC, Puah SM, Chua KH, Chew CH. Staphylococcus aureus infections in Malaysia: a review of antimicrobial resistance and characteristics of the clinical isolates, 1990-2017. Antibiotics. 2019;8(3):128-56.

10. Mehrotra M, Wang G, Johnson WM. Multiplex PCR for detection of genes for Staphylococcus aureus enterotoxins, exfoliative toxins, toxic shock syndrome toxin 1, and methicillin resistance. J Clin Microbiol. 2000;38(3): 1032-5.

11. Lakhundi S, Zhang K. Methicillin-resistant Staphylococcus aureus: molecular characterization, evolution, and epidemiology. Clin Microbiol Rev. 2018; 31(4):1-103.

12. Dweba CC, Zishiri O, El Zowalaty M. Methicillin-resistant Staphylococcus aureus: livestock-associated, antimicrobial, and heavy metal resistance. Infect Drug Resist. 2018;11:2497-509.

13. Diaz R, Afreixo V, Ramalheira E, Rodrigues C, Gago B. Evaluation of vancomycin MIC creep in methicillin-resistant Staphylococcus aureus infections-a systematic review and meta-analysis. Clin Microbiol Infect. 2018, 24(2):97-104.

14. Chung Y, Kim TS, Min YG, Hong YJ, Park JS, Hwang SM, Song K, Kim ES, Park $\mathrm{KU}$, Kim HB, et al. Usefulness of multiplex real-time PCR for simultaneous pathogen detection and resistance profiling of Staphylococcal bacteremia. Biomed Res Int. 2016;2016:6913860-7.

15. Pereira LA, Harnett GB, Hodge MM, Cattell JA, Speers DJ. Real-time PCR assay for detection of blaZ genes in Staphylococcus aureus clinical isolates. J Clin Microbiol. 2014;52(4):1259-61.

16. Kim DW, Kilgore PE, Kim EJ, Kim SA, Anh DD, Dong BQ, Kim JS, Seki M. The enhanced pneumococcal LAMP assay: a clinical tool for the diagnosis of meningitis due to Streptococcus pneumoniae. PLoS One. 2012;7(8):e42954.

17. Ge B, Domesle KJ, Yang Q, Hammack TS, Wang SS, Deng X, Hu L, Zhang G, $\mathrm{Hu} Y$, Lai $X$, et al. Multi-laboratory validation of a loop-mediated isothermal amplification method for screening Salmonella in animal food. Front Microbiol. 2019:10:1-13.

18. Li S, Liu Y, Wang Y, Chen H, Liu C, Wang Y. Lateral flow biosensor combined with loop-mediated isothermal amplification for simple, rapid, sensitive, and reliable detection of Brucella spp. Infect Drug Resist. 2019;12:2343-53.

19. Waterfield T, Fairley D, Lynn F, Blackwood B, Shields MD. A protocol for a systematic review of the diagnostic accuracy of loop-mediated-isothermal 
AMPlification (LAMP) in diagnosis of invasive meningococcal disease in children. Systematic Reviews. 2018;7(1):86-90.

20. Seki M, Kilgore PE, Kim EJ, Ohnishi M, Hayakawa S, Kim DW. Loop-mediated isothermal amplification methods for diagnosis of bacterial meningitis. Front Pediatr. 2018;6:57.

21. Yang Q, Domesle KJ, Wang F, Ge B. Rapid detection of Salmonella in food and feed by coupling loop-mediated isothermal amplification with bioluminescent assay in real-time. BMC Microbiol. 2016;16:112.

22. Wang Y, Wang Y, Ma A, Li D, Luo L, Liu D, Jin D, Liu K, Ye C. Rapid and sensitive isothermal detection of nucleic-acid sequence by multiple cross displacement amplification. Sci Rep. 2015;5:11902.

23. Jiao W, Wang Y, Wang G, Wang Y, Xiao J, Sun L, Li J, Wen S, Zhang T, Ma Q, et al. Development and clinical validation of multiple cross displacement amplification combined with nanoparticles-based biosensor for detection of Mycobacterium tuberculosis: preliminary results. Front Microbiol. 2019;10:2135.

24. Wang Y, Li H, Wang Y, Zhang L, Xu J, Ye C. Loop-mediated isothermal amplification label-based gold nanoparticles lateral flow biosensor for detection of Enterococcus faecalis and Staphylococcus aureus. Front Microbiol. 2017;8:192.

25. Grousd JA, Rich HE, Alcorn JF. Host-pathogen interactions in gram-positive bacterial pneumonia. Clin Microbiol Rev. 2019;32(3):e00107.

26. Galar A, Weil AA, Dudzinski DM, Muñoz P, Siedner MJ. Methicillin-resistant Staphylococcus aureus prosthetic valve endocarditis: pathophysiology, epidemiology, clinical presentation, diagnosis, and management. Clin Microbiol Rev. 2019;32(2):e00041.

27. Hassoun A, Linden PK, Friedman B. Incidence, prevalence, and management of MRSA bacteremia across patient populations-a review of recent developments in MRSA management and treatment. Crit Care. 2017;21:211.

28. Balasubramanian D, Harper L, Shopsin B, Torres VJ. Staphylococcus aureus pathogenesis in diverse host environments. Pathog Dis. 2017;75(1):ftx005.

29. Cosimi RA, Beik N, Kubiak DW, Johnson JA. Ceftaroline for severe methicillinresistant Staphylococcus aureus infections: a systematic review. Open Forum Infect Dis. 2017;4(2):ofx084.

30. Aires-de-Sousa M. Methicillin-resistant Staphylococcus aureus among animals: current overview. Clin Microbiol Infect. 2017;23(6):373-80

31. Holland TL, Arnold C, Fowler VG. Clinical management of Staphylococcus aureus bacteremia. JAMA. 2014;312(13):1330

32. Hallin M, Maes N, Byl B, Jacobs F, De Gheldre Y, Struelens MJ. Clinical impact of a PCR assay for identification of Staphylococcus aureus and determination of methicillin resistance directly from blood cultures. J Clin Microbiol. 2003;41(8):3942-4.

33. Al-Talib H, Yean CY, Al-Khateeb A, Hasan H, Ravichandran M. Rapid detection of methicillin-resistant Staphylococcus aureus by a newly developed dry reagent-based polymerase chain reaction assay. J Microbiol Immunol Infect. 2014;47(6):484-90.

34. Hu S, Niu L, Zhao F, Yan L, Nong J, Wang C, Gao N, Zhu X, Wu L, Bo T, et al. Identification of Acinetobacter baumannii and its carbapenem-resistant gene

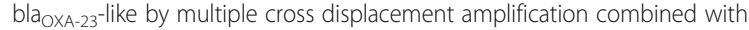
lateral flow biosensor. Sci Rep. 2019;9:17888.

35. Cheng X, Yang J, Wang M, Wu P, Du Q, He J, Tang Y. Visual and rapid detection of Acinetobacter baumannii by a multiple cross displacement amplification combined with nanoparticles-based biosensor assay. AMB Express. 2019;9(1):30.

\section{Publisher's Note}

Springer Nature remains neutral with regard to jurisdictional claims in published maps and institutional affiliations.

\section{Ready to submit your research? Choose BMC and benefit from}

- fast, convenient online submission

- thorough peer review by experienced researchers in your field

- rapid publication on acceptance

- support for research data, including large and complex data types

- gold Open Access which fosters wider collaboration and increased citations

- maximum visibility for your research: over $100 \mathrm{M}$ website views per year

At BMC, research is always in progress.

Learn more biomedcentral.com/submissions 